\title{
Tauroursodeoxycholic acid reduces the invasion of MDA-MB-231 cells by modulating matrix metalloproteinases 7 and 13
}

\author{
GA-YOUNG PARK ${ }^{1,2^{*}}$, YU KYEONG HAN ${ }^{1 *}$, JEONG YOON HAN $^{1}$ and CHANG GEUN LEE ${ }^{1}$ \\ ${ }^{1}$ Research Center, Dongnam Institute of Radiological and Medical Sciences, Busan 619-953; ${ }^{2}$ Department of Molecular Biology, \\ College of Natural Sciences, Pusan National University, Busan 609-735, Republic of Korea
}

Received March 30, 2015; Accepted June 17, 2016

DOI: $10.3892 / \mathrm{ol} .2016 .4842$

\begin{abstract}
Tauroursodeoxycholic acid (TUDCA) is a conjugated form of UDCA that modulates several signaling pathways and acts as a chemical chaperone to relieve endoplasmic reticulum (ER) stress. The present study showed that TUDCA reduced the invasion of the MDA-MB-231 metastatic breast cancer cell line under normoxic and hypoxic conditions using an in vitro invasion assay. Quantitative polymerase chain reaction assay revealed that the reduced invasion following TUDCA treatment was associated with a decreased expression of matrix metalloproteinase (MMP)-7 and -13, which play important roles in invasion and metastasis. Inhibitors and short hairpin RNAs were used to show that the effect of TUDCA in the reduction of invasion appeared to be dependent on the protein kinase RNA-like ER kinase pathway, a downstream ER stress signaling pathway. Thus, TUDCA is a candidate anti-metastatic agent to target the ER stress pathway.
\end{abstract}

\section{Introduction}

The endoplasmic reticulum (ER) is the central compartment for producing and folding cell surface receptors and secreted proteins. The ER is also involved in calcium balance regulation and the biosynthesis of cholesterol and steroids (1). Perturbations in ER homeostasis lead to the accumulation of misfolded proteins, which activates an adaptation response called the ER stress response, also known as the unfolded protein response (2). The ER stress response is mediated by three main pathways: The inositol-requiring enzyme 1 (IRE1), protein kinase RNA-like ER kinase (PERK) and activating

Correspondence to: Dr Chang Geun Lee, Research Center, Dongnam Institute of Radiological and Medical Sciences, 40 Jwadong-gil, Jangan-eup, Gijang-gun, Busan 619-953, Republic of Korea

E-mail: cglee@dirams.re.kr

*Contributed equally

Key words: tauroursodeoxycholic acid, endoplasmic reticulum stress, invasion, matrix metalloproteinase, protein kinase RNA-like endoplasmic reticulum kinase transcription factor 6 (ATF6) pathways. The activation of these pathways is associated with stress relief and ER function restoration (3).

Cancer cells are associated with various stressors, such as hypoxia, nutrient deprivation and $\mathrm{pH}$ changes, which activate cellular stress response pathways, including the ER stress response (4). The ER stress response is therefore crucial to cancer progression and other cancer cell phenotypes, including invasion and migration. Li et al (5) showed that activating ER stress in breast cancer cells through use of Adriamycin or Tunicamycin enhances the invasion and migration associated with heparinase. Hypoxia-enhanced migration of breast cancer cells occurs through the PERK-ATF4 pathway during the ER stress response (6). These results suggest that activating ER stress in breast cancer cells enhances their invasion and migration. The ER stress pathway is activated in breast cancer cells even in the absence of external stress, and the activation status is associated with cancer growth, relapse and maintenance of a cancer stem cell population (7).

Tauroursodeoxycholic acid (TUDCA) is a taurine conjugated form of UDCA that has been used to treat jaundice in Asian countries and has been approved by the US Food and Drug Administration for treating primary biliary cirrhosis (8). TUDCA acts as a chemical chaperone and modulates several signaling pathways (9).

The present study investigated whether basal ER stress is associated with the invasion and migration of breast cancer using TUDCA as a chemical chaperone. TUDCA reduced the invasiveness of the MDA-MB-231 metastatic breast cancer cell line under normoxic and hypoxic conditions. In addition, the PERK pathway appeared to be involved in cancer cell invasion when using inhibitors and short hairpin RNAs (shRNAs). These results suggested that the ER stress pathway may serve as a therapeutic target for the development of anti-metastatic drugs and chemical chaperones, such as TUDCA, may be candidates for anti-metastatic agents.

\section{Materials and methods}

Cell culture. MDA-MB-231 cells were purchased from the American Type Culture Collection (Manassas, VA, USA). Cell culture and transfection were performed as previously described (10). Sense and antisense oligonucleotides for shRNAs targeting human PERK-299 (5'-GCGGCAGGTCAT 
TAGTAATTA-3') and PERK-506 (5'-GCATGGAAACAG TTCCTTTCA-3') were generated, annealed and cloned into the pSUPER.puro vector (Oligoengine, Seattle, WA, USA), according to the manufacturer's instructions. For transient transfections of shRNAs, MDA-MB-231 cells were electroporated using Neon ${ }^{\circledR}$ Transfection System (Thermo Fisher Scientific Inc., Waltham, MA, USA), according to the manufacturer's protocol. TUDCA (Sigma-Aldrich, St. Louis, MO, USA), an ATF6 inhibitor [4-(2-aminoethyl)benzenesulfonyl fluoride hydrochloride], an IRE1 inhibitor (4u8C) and a PERK inhibitor (GSK2606414) (Calbiochem, San Diego, CA, USA) were used. Cell viability was evaluated using the ADAM-MC Automatic Cell Counter (NanoEnTek, Inc., Seoul, Korea). An MTT assay was performed using the CellTiter 96 Non-Radioactive Cell Proliferation Assay kit (Promega, Madison, WI, USA), according to the manufacturer's instructions.

Hypoxia treatment. Cells were exposed to hypoxia using an anaerobic system (Thermo Scientific, Inc., Marietta, $\mathrm{OH}$, USA) using mixed gas $\left(1 \% \mathrm{O}_{2}, 5 \% \mathrm{CO}_{2}, \mathrm{~N}_{2}\right.$ balance). Oxygen concentration was checked with an $\mathrm{O}_{2}$ sensor (New Cosmos, Osaka, Japan) prior to hypoxia treatment. Cells were kept in $37^{\circ} \mathrm{C}$ incubation chamber in an anaerobic system.

Invasion assay. Upper membranes of cell culture inserts (BD Biosciences, Franklin Lakes, NJ, USA) were coated with Matrigel diluted in Opti-MEM ${ }^{\circledR}$ Reduced Serum Medium (1:10 ratio; Thermo Fisher Scientific, Inc.) for $1.5 \mathrm{~h}$ and then rehydrated with serum-free Dulbecco's modified Eagle's medium (DMEM) for $1 \mathrm{~h}$. Next, $2.5 \times 10^{4}$ cells suspended in serum-free DMEM were seeded into the upper layer of cell culture inserts, and DMEM with $10 \%$ fetal bovine serum was added to the lower chambers for $24 \mathrm{~h}$ at $37^{\circ} \mathrm{C}$. The inserts were then removed from the medium, fixed in $100 \%$ methanol and stained with $0.1 \%$ crystal violet dye for $10 \mathrm{~min}$. The upper surfaces of the inserts were wiped with swaps, and the membranes were isolated from the inserts to prepare slides. The invaded cells were observed and images were captured using an Eclipse 80i Upright microscope (Nikon, Tokyo, Japan) and the Image-Pro Plus software (Media Cybernetics Inc., Rockville, MD, USA).

Wound-healing assay. The MDA-MB-231 cells were treated with either the vehicle control (EtOH) or TUDCA, and their migration ability was compared using the wound-healing assay. Briefly, $1 \times 10^{5}$ cells were plated into 6 -well plates. Following $24 \mathrm{~h}$ of incubation, the cell monolayers were scratched using a $200-\mu 1$ pipette tip, and the medium was changed for fresh medium containing the vehicle control $(\mathrm{EtOH})$ or $0.5 \mathrm{mM}$ TUDCA. The widths of the scratches were monitored for $54 \mathrm{~h}$, and images were captured at $0,6,24$ and $54 \mathrm{~h}$ using a Nikon Eclipse TS100 (Nikon) inverted microscope and the Image-Pro Plus software.

RNA purification, $C D N A$ synthesis and reverse transcription-quantitative polymerase chain reaction (RT-qPCR). Total cellular RNA was extracted using the RNeasy Mini kit (Qiagen, Valencia, CA, USA), according to the manufacturer's instructions. RNA $(1 \mu \mathrm{g})$ was used for cDNA synthesis using the AccuPower RT PreMix and oligo(dT) primers (Bioneer,
Daejeon, South Korea). RT-qPCR was performed with the cDNA, Maxima SYBR Green qPCR Master Mix (Thermo Fisher Scientific, Inc.) and the specific primers listed below using the CFX96 Real-Time System (Bio-Rad, Hercules, CA, USA). The primer sequences were as follows: MMP-7 forward, 5'-GTATGGGACATTCCTCTGATCC-3' and reverse, 5'-CCA ATGAATGAATGAATGGATG-3'; MMP-13 forward, 5'-AAC CAGGTCTGGAGATATGATGA-3' and reverse, 5'-TGTATG GGTCCGTTGAAAAA-3'; and GAPDH forward, 5'-GAA ATCCCATCACCATCTTCCAGG-3' and reverse, 5'-GAG CCCCAGCCTTCTCCATG-3'. The qPCR parameters were 5 min at $95^{\circ} \mathrm{C}$, followed by 40 cycles of $10 \mathrm{sec}$ at $95^{\circ} \mathrm{C}, 10 \mathrm{sec}$ at $60^{\circ} \mathrm{C}$ and $10 \mathrm{sec}$ at $70^{\circ} \mathrm{C}$. A melting curve step $\left(65-95^{\circ} \mathrm{C}\right.$ at increments of $0.5^{\circ} \mathrm{C}$ ) was performed at the end of the qPCR. Relative quantification of target gene expression was calculated by the quantitative threshold cycle (Cq) method, using GAPDH as an endogenous reference gene for normalization (11). RT-qPCR was performed $>3$ times for each gene and representative results are shown.

Statistical analysis. Results are expressed as the mean \pm standard deviation. Statistical significance was analyzed using the SPSS version 18 software for Windows (SPSS Inc., Chicago, IL, USA). Significant differences between two groups were evaluated by Student's t-test. $\mathrm{P}<0.05$ was considered to indicate a statistically significant difference.

\section{Results}

TUDCA reduces invasion by $M D A-M B-231$ breast cancer cells. In the present study, TUDCA was used as a chemical chaperone to investigate the role of ER stress in breast cancer cell invasiveness. MDA-MB-231 cells were treated with TUDCA for $16 \mathrm{~h}$ and then used in the invasion assay with Matrigel-coated membranes. TUDCA did not significantly modulate cell viability, however, it did significantly reduce MDA-MB-231 cell invasion $\left(36.6 \%\right.$; $\mathrm{P}=2.122 \times 10^{-6}$ vs. vehicle; Fig. 1). TUDCA did not significantly modulate the migration or density of the MDA-MB-231 cells following a 54-h incubation period (Fig. 2). These results suggested that the reduced invasion following the TUDCA treatment was not due to reduced migration or proliferation.

Reduced invasion due to TUDCA is associated with reduced expression of MMP-7 and -13. Several molecules associated with invasion were tested using western blotting and RT-qPCR. the expression of MMP-7 and -13 was found to be significantly decreased following treatment with TUDCA (38.4\% and $24.7 \%$, respectively; $\mathrm{P}=5.863 \times 10^{-6}$ and $\mathrm{P}=1.006 \times 10^{-5}$ vs. vehicle, respectively; Fig. 3 ), suggesting that TUDCA may reduce invasion by decreasing MMP-7 and -13 expression. As cancer cell invasion is associated with hypoxia, the possibility of TUDCA modulating invasion under hypoxic conditions was also investigated. Consistent with the normoxic result, TUDCA significantly decreased MDA-MB-231 cell invasion (28.9\%; $\mathrm{P}=8.971 \times 10^{-7}$ vs. vehicle; Fig. $\left.4 \mathrm{~A}\right)$. Furthermore, the decreased invasion was shown to be associated with decreased MMP-7 and -13 expression under hypoxic conditions (41.1\% and $28.7 \%$, respectively; $\mathrm{P}=1.668 \times 10^{-4}$ and $\mathrm{P}=3.569 \times 10^{-4}$ vs. vehicle, respectively; Fig. 4B). 

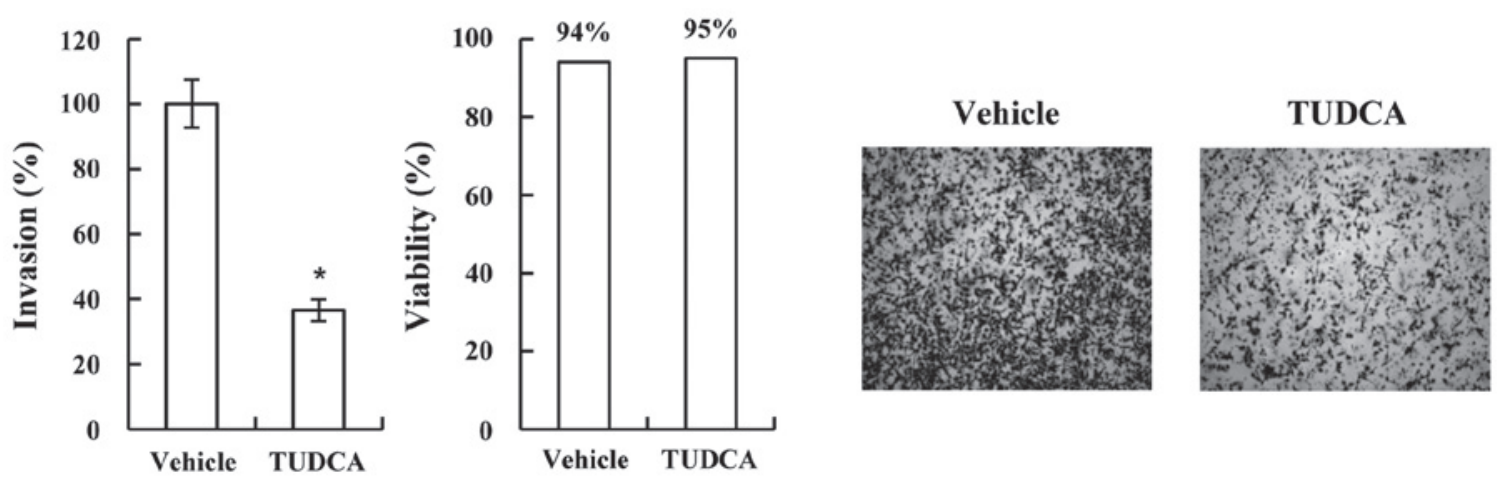

Figure 1. TUDCA reduced breast cancer MDA-MB-231 cell invasion without affecting cell viability. MDA-MB-231 cells were treated with $0.5 \mathrm{mM}$ TUDCA for $16 \mathrm{~h}$ and were analyzed using an invasion assay. The number of cells invading the Matrigel was normalized against vehicle control cells invading the Matrigel to $100 \%$. Cell viability following vehicle or TUDCA treatment is shown. Representative invasion assay images are shown. Data are presented as the mean \pm standard deviation. "P<0.000005 vs. vehicle. TUDCA, tauroursodeoxycholic acid.
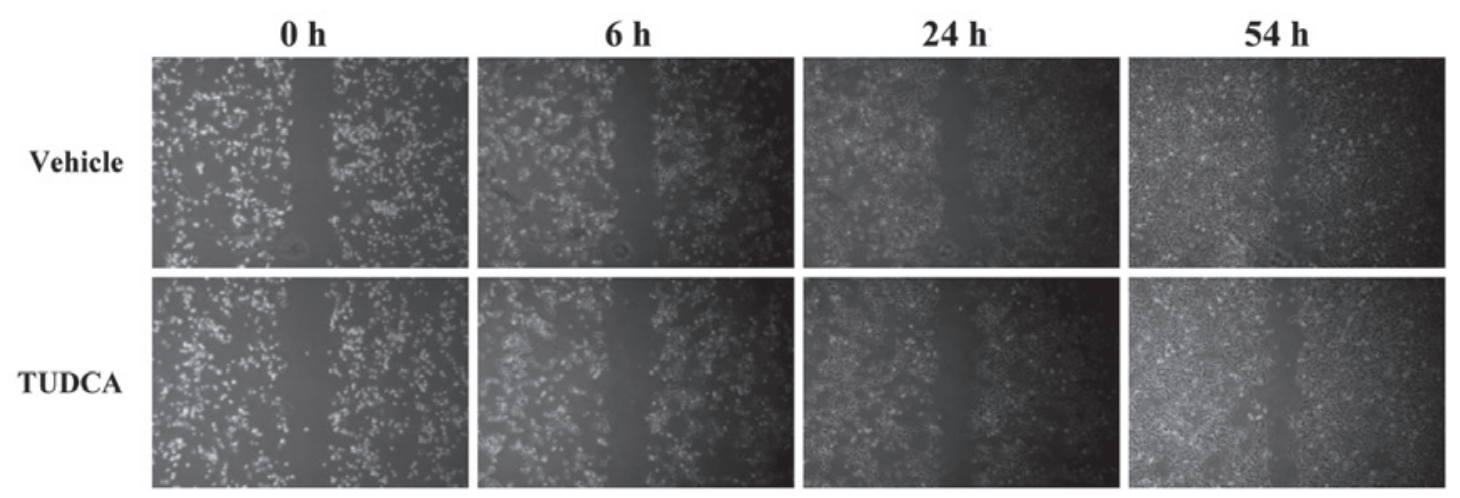

Figure 2. TUDCA does not modulate MDA-MB-231 breast cancer cell migration. A wound-healing assay was performed to evaluate MDA-MB-231 cell migration during treatment with vehicle or $0.5 \mathrm{mM}$ TUDCA. Images were captured at the indicated time points. TUDCA, tauroursodeoxycholic acid.

MMP-7

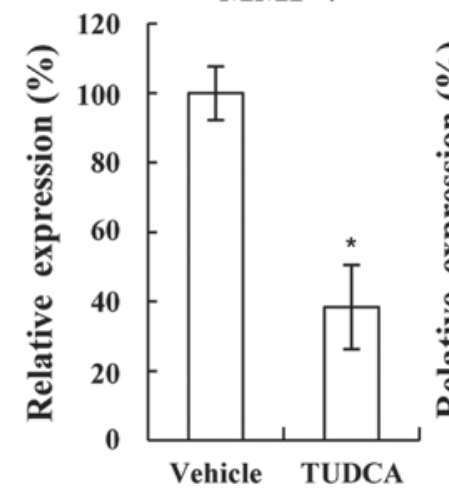

MMP-13

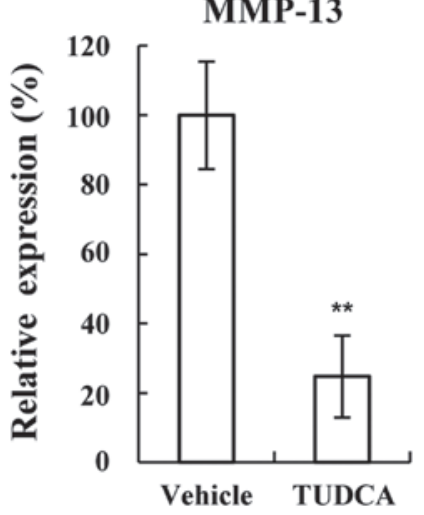

Figure 3. Reduced MDA-MB-231 breast cancer cell invasion in response to TUDCA is associated with reduced MMP-7 and -13 expression. MDA-MB-231 cells were treated with $0.5 \mathrm{mM}$ TUDCA, total RNA was purified and cDNA was synthesized. Reverse transcription-quantitative polymerase chain reaction was performed using SYBR Green. mRNA levels were normalized to those of glyceraldehyde-3-phosphate dehydrogenase and plotted by setting the expression of the vehicle control to $100 \%$. Data are presented as the mean \pm standard deviation. ${ }^{*} \mathrm{P}<0.00001$ and ${ }^{* *} \mathrm{P}<0.00005$ vs. vehicle. MMP, matrix metalloproteinase; TUDCA, tauroursodeoxycholic acid.

PERKin the ERstresspathwayisassociated with MDA-MB-231 cell invasion. The ER stress pathway includes three downstream pathways: ATF6, IRE1 and PERK. The present study used inhibitors of these downstream pathways to investigate which pathway is involved in ER stress-mediated invasion. Only the PERK inhibitor significantly decreased the invasion of the MDA-MB-231 cells (75.9\%; $\mathrm{P}=1.197 \times 10^{-3}$ vs. vehicle;
Fig. 5A). As the PERK inhibitor may have inhibited other signaling pathways, shRNAs targeting PERK were used to confirm the results. shRNAs targeting different PERK mRNA regions were prepared and used in the invasion assay. Consistent with the results of the use of the inhibitor, depleting PERK with the shRNAs was found to decrease MDA-MB-231 cell invasion (64.7\%; P=7.961 $110^{-4}$ vs. vehicle; Fig. 5B). These 
A

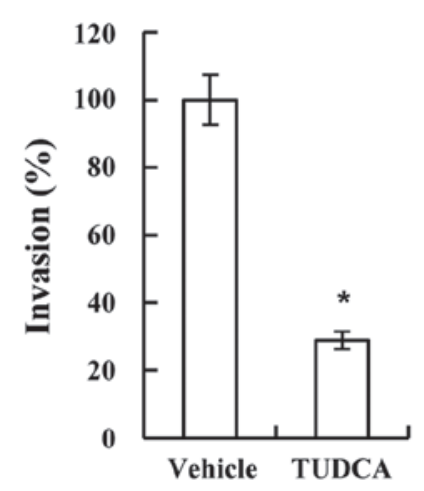

Vehicle

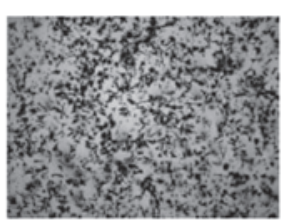

TUDCA

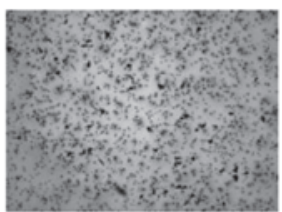

B

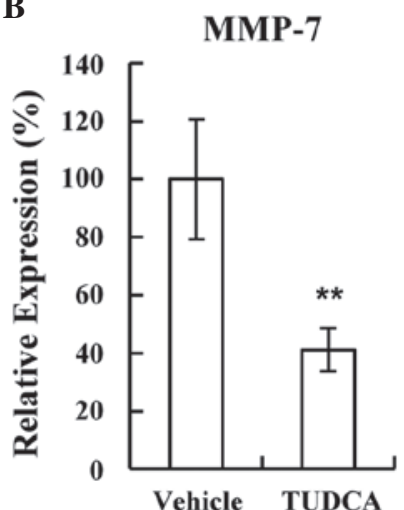

MMP-13

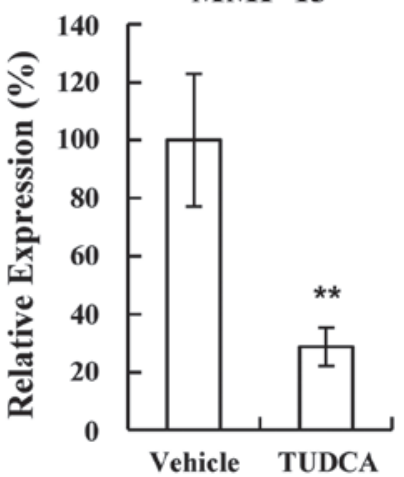

Figure 4. TUDCA reduces MDA-MB-231 breast cancer cell invasion under hypoxic conditions. (A) MDA-MB-231 cells were treated with 0.5 mM TUDCA for $16 \mathrm{~h}$ under hypoxic conditions $\left(\sim 1 \% \mathrm{O}_{2}\right)$ and used in the invasion assay. The number of cells invading the Matrigel was normalized by setting the number of vehicle control cells invading the Matrigel to $100 \%$. Representative invasion assay images are shown. (B) Total RNA was purified, and complementary DNA synthesized. Reverse transcription-quantitative polymerase chain reaction was performed using SYBR Green. mRNA levels were normalized to those of glyceraldehyde-3-phosphate dehydrogenase and plotted by setting the expression of the vehicle control to $100 \%$. Data are presented as the mean \pm standard deviation. ${ }^{*} \mathrm{P}<0.000001$ and ${ }^{* *} \mathrm{P}<0.0005$ vs. vehicle. MMP, matrix metalloproteinase; TUDCA, tauroursodeoxycholic acid.

A

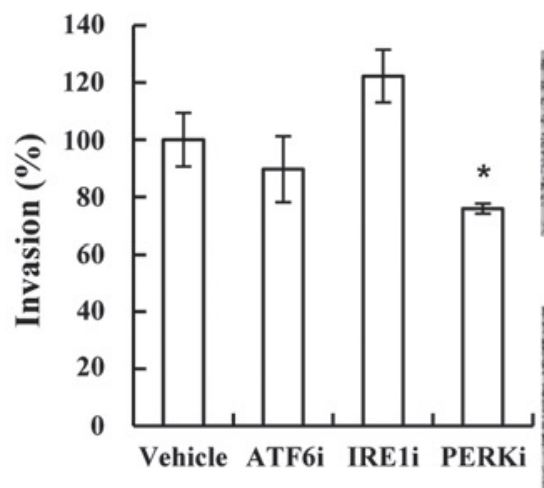

Vehicle

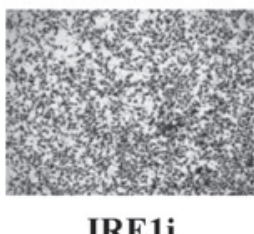

IRE1i

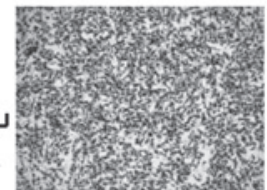

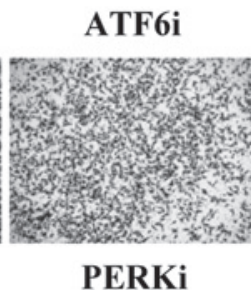

PERKi

B
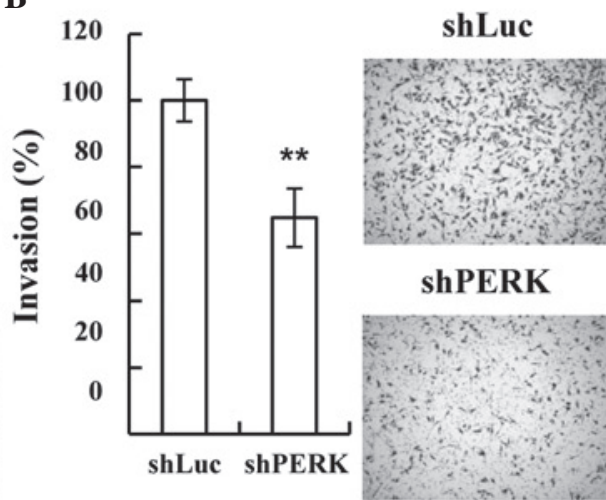

Figure 5. PERK in the endoplasmic reticulum stress pathway is involved in MDA-MB-231 breast cancer cell invasion. (A) MDA-MB-231 cells were incubated with the indicated inhibitors (300 $\mu \mathrm{M}$ ATFi, $50 \mu \mathrm{M}$ IREli and $10 \mu \mathrm{M}$ PERKi) and used in the Matrigel-based invasion assay. The same number of cells was plated on a 24 -well plate and used in the MTT assay to evaluate cell viability, which was used for normalization. The number of cells invading the Matrigel was normalized by setting the number of vehicle control cells invading the Matrigel to $100 \%$. Representative invasion assay images are shown. Data are presented as the mean \pm standard deviation. ${ }^{*} \mathrm{P}<0.005$ for PERKi vs. vehicle. (B) shLuc and shPERK were transfected into MDA-MB-231 cells and used in the Matrigel-based invasion assay. The same number of cells was plated on a 24-well plate and used in the MTT assay to evaluate cell viability, which was used for normalization. The number of cells invading the Matrigel was normalized by setting the number of vehicle control cells invading the Matrigel to 100\%. Representative invasion assay images are shown. Data are presented as the mean \pm standard deviation. ${ }^{* *} \mathrm{P}<0.001$ vs. shLuc. ATF6i, activating transcription factor 6 inhibitor; PERKi, protein kinase RNA-like endoplasmic reticulum kinase inhibitor; IREi, inositol-requiring enzyme 1 inhibitor; shRNA, short hairpin RNA; shLuc, control shRNA; shPERK, shRNA targeting PERK mRNA.

results suggested that the PERK pathway may be involved in the invasiveness of metastatic breast cancer.

\section{Discussion}

MMPs are a family of zinc-dependent endopeptidases that are used in extracellular matrix remodeling and are associated with embryogenic tissue remodeling, and angiogenic and pathological processes, such as cancer cell invasion and arthritis (12). Cancer cells detach from primary cancers and invade through the basement membrane and extracellular matrix cleaved by MMPs. The present study found that the expression of MMP-7 and -13 decreased significantly following TUDCA treatment. These MMPs are involved in cell invasion in gastric cancer and Kaposi's sarcoma $(13,14)$. ER stress activation in breast cancer cells using Adriamycin has been shown to enhance invasion by activating heparinase (5); however, this is the first study to report the involvement of the ER stress pathway in the regulation of MMP-7 and -13 during breast cancer cell invasion under basal conditions without an external stimulus-activated ER stress response.

The majority of solid tumors have hypoxic regions, due to impaired angiogenesis. Hypoxia is associated with metastasis, particularly in patients with hypoxic tumors (15-17). The present study showed that TUDCA reduced the invasion of MDA-MB-231 cells under hypoxic conditions. Hypoxia has been shown to activate the ER stress pathway in cancer cells $(18,19)$. Therefore, TUDCA may decrease the ER stress response activated by hypoxia, resulting in reduced invasion.

In the present study, TUDCA was shown to act as a chemical chaperone that reduces the invasion of the MDA-MB-231 metastatic breast cancer cell line by decreasing the basal ER 
stress response, suggesting that the ER stress pathway may be involved in breast cancer cell invasion, as well as survival against stressors such as hypoxia, glucose starvation and $\mathrm{pH}$ changes. Furthermore, TUDCA was found to reduce breast cancer cell invasion under hypoxic conditions, which suggested that the ER stress pathway may be a good therapeutic target for cancer metastasis, and that chemical chaperones, such as TUDCA, may be useful for that purpose. TUDCA is not currently approved by the Food and Drug Administration, but UDCA, which is approved, is metabolized to TUDCA in the liver.

\section{Acknowledgements}

This study was supported by the National Research Foundation of Korea (DIRAMS) grant funded by the Korea government (MSIP) (grant nos. 50597-2014 and 50491-2015).

\section{References}

1. Hetz C, Martinon F, Rodriguez D and Glimcher LH: The unfolded protein response: Integrating stress signals through the stress sensor IRE1 $\alpha$. Physiol Rev 91: 1219-1243, 2011.

2. Walter P and Ron D: The unfolded protein response: From stress pathway to homeostatic regulation. Science 334: 1081-1086, 2011.

3. Lin JH, Walter P and Yen TS: Endoplasmic reticulum stress in disease pathogenesis. Annu Rev Pathol 3: 399-425, 2008.

4. Healy SJ, Gorman AM, Mousavi-Shafaei P, Gupta S and Samali A: Targeting the endoplasmic reticulum-stress response as an anticancer strategy. Eur J Pharmacol 625: 234-246, 2009.

5. Li Y, Liu H, Huang YY, Pu LJ, Zhang XD, Jiang CC and Jiang ZW: Suppression of endoplasmic reticulum stress-induced invasion and migration of breast cancer cells through the downregulation of heparanase. Int J Mol Med 31: 1234-1242, 2013

6. Nagelkerke A, Bussink J, Mujcic H, Wouters BG, Lehmann S, Sweep FC and Span PN: Hypoxia stimulates migration of breast cancer cells via the PERK/ATF4/LAMP3-arm of the unfolded protein response. Breast Cancer Res 15: R2, 2013.

7. Chen X, Iliopoulos D, Zhang Q, Tang Q, Greenblatt MB, Hatziapostolou M, Lim E, Tam WL, Ni M, Chen Y, et al: XBP1 promotes triple-negative breast cancer by controlling the HIF1a pathway. Nature 508: 103-107, 2014.
8. Wimmer R, Hohenester S, Pusl T, Denk GU, Rust C and Beuers U: Tauroursodeoxycholic acid exerts anticholestatic effects by a cooperative cPKC alpha-/PKA-dependent mechanism in rat liver. Gut 57: 1448-1454, 2008

9. Vang S, Longley K, Steer CJ and Low WC: The unexpected uses of urso- and tauroursodeoxycholic acid in the treatment of non-liver diseases. Glob Adv Health Med 3: 58-69, 2014.

10. Han YK, Lee JH, Park GY, Chun SH, Han JY, Kim SD, Lee J, Lee CW, Yang K and Lee CG: A possible usage of a CDK4 inhibitor for breast cancer stem cell-targeted therapy. Biochem Biophys Res Commun 430: 1329-1333, 2013.

11. Livak KJ and Schmittgen TD: Analysis of relative gene expression data using real-time quantitative PCR and the 2(-Delta Delta C(T)) Method. Methods 25: 402-408, 2001.

12. Yadav L, Puri N, Rastogi V, Satpute P, Ahmad R and Kaur G: Matrix metalloproteinases and cancer-roles in threat and therapy. Asian Pac J Cancer Prev 15: 1085-1091, 2014.

13. Ye Y, Zhou X, Li X, Tang Y, Sun Y and Fang J: Inhibition of epidermal growth factor receptor signaling prohibits metastasis of gastric cancer via downregulation of MMP7 and MMP13. Tumour Biol 35: 10891-10896, 2014.

14. Zhang J, Wang S, Lu L and Wei G: MiR99a modulates MMP7 and MMP13 to regulate invasiveness of Kaposi's sarcoma. Tumour Biol 35: 12567-12573, 2014.

15. Chia SK, Wykoff CC, Watson PH, Han C, Leek RD, Pastorek J, Gatter KC, Ratcliffe P and Harris AL: Prognostic significance of a novel hypoxia-regulated marker, carbonic anhydrase IX, in invasive breast carcinoma. J Clin Oncol 19: 3660-3668, 2001.

16. Hockel M, Schlenger K, Aral B, Mitze M, Schaffer U and Vaupel P: Association between tumor hypoxia and malignant progression in advanced cancer of the uterine cervix. Cancer Res 56: 4509-4515, 1996.

17. Rademakers SE, Span PN, Kaanders JH, Sweep FC, van der Kogel AJ and Bussink J: Molecular aspects of tumour hypoxia. Mol Oncol 2: 41-53, 2008

18. Liu Y, László C, Liu Y, Liu W, Chen X, Evans SC and Wu S: Regulation of G(1) arrest and apoptosis in hypoxia by PERK and GCN2-mediated eIF2alpha phosphorylation. Neoplasia 12: 61-68, 2010

19. Rouschop KM, Dubois LJ, Keulers TG, van den Beucken T, Lambin P, Bussink J, van der Kogel AJ, Koritzinsky M and Wouters BG: PERK/eIF2 $\alpha$ signaling protects therapy resistant hypoxic cells through induction of glutathione synthesis and protection against ROS. Proc Natl Acad Sci USA 110: 4622-4627, 2013. 